\title{
8-Iso-Prostaglandin F2 Alpha to Creatinine Ratio Measurement
}

National Cancer Institute

\section{Source}

National Cancer Institute. 8-Iso-Prostaglandin F2 Alpha to Creatinine Ratio

Measurement. NCI Thesaurus. Code C119291.

The determination of the ratio of the 8-iso-prostaglandin F2 alpha to creatinine in a biological specimen. 